\title{
A propósito de una verdad contrahegemónica: aprendizajes desde la decisión de la Corte Penal Internacional sobre el ataque contra la flotilla de la libertad ${ }^{1}$
}

About a counter-hegemonic truth: Learnings from the decision of the International Criminal Court on the attack against the freedom flotilla

Sandra Rocío Gamboa Rubiano ${ }^{2}$

Fecha de recepción: 20 de enero de 2016

Fecha de aprobación: 7 de marzo de 2016

\section{RESUMEN}

Este artículo indaga sobre las implicaciones contrahegemónicas del ejercicio del derecho a la verdad en el proceso penal. Ello tiene lugar tras admitirse su trascendencia en regímenes normativos distintos, pero complementarios, como el Derecho Internacional de los Derechos Humanos y el Derecho Penal Internacional. Para tales efectos, se concentra en el análisis de las posibilidades de una verdad contrahegemónica que vehicule la verdad histórica de las víctimas hacia los procesos penales, a partir de la reciente decisión de la Corte Penal Internacional (CPI) sobre el ataque contra la "Flotilla de la Libertad".

Palabras clave: Corte Penal Internacional, procesos penales, verdad contrahegemónica, víctimas.

1 Artículo de investigación científica dentro del proyecto: "Dimensiones del Derecho Internacional de los Derechos Humanos en la Justicia Transicional, para ejercer los derechos a la verdad y a la justicia en procesos penales ordinarios sobre graves violaciones de derechos humanos: El papel de la Defensa de Familia". Código del Grupo: COL0081737. Grupo de investigación de Familia, Conflictos Sociales y Proyección Social de la Facultad de Derecho de la Universidad La Gran Colombia. Categoría B. Investigadora responsable: Sandra Gamboa Rubiano PhD.

2 Abogada de la Universidad Nacional de Colombia. Doctora en Derecho y magíster en Defensa de los Derechos Humanos y el Derecho Internacional Humanitario ante organismos, tribunales y cortes internacionales, por la Universidad Santo Tomás. Docente investigadora adscrita a la Facultad de Derecho de la Universidad La Gran Colombia. Correo Electrónico: sandra.gamboa.rubiano@gmail.com 


\begin{abstract}
This article explores the counter-hegemonic implications in exercising the right to truth for victims in criminal proceedings, having acknowledged its importance in different regulatory regimes such as International Human Rights Law, and International Criminal Law. To this end, it focuses on the analysis of the possibilities of a counter-hegemonic truth that conveys the historical truth of victims to criminal proceedings, based on the recent decision by the International Criminal Court regarding the attack on the "Freedom Flotilla".
\end{abstract}

Keywords: Counter-hegemonic truth, criminal proceedings, International Criminal Court, victims.

“Cuando se retiran los cadáveres, empieza la política: así es (así parece ser) tanto en Hamlet como en Antígona: Fortinbras o Creonte vienen a restaurar el orden justo de la Polis, amenazado por el 'estado de naturaleza' y la guerra de todos contra todos. Pero, desde luego, esto podría ser tan sólo una ilusión retrospectiva, un efecto de lectura retardado, generado por las 'fuerzas reactivas' -en el sentido nietzscheano- de las modernas filosofías contractualistas (todavía, o de nuevo, dominantes tanto en la academia como en el sentido común político de hoy), que se distraen con prolijidad y empeño ante la verdad histórica evidente de que todo 'orden justo' instaurado por un 'contrato' es, no sólo pero también, el resultado de la victoria de una de las partes en una relación de fuerzas; que la 'universalidad' del consenso es el reconocimiento (no necesariamente consciente) de la hegemonía de un partido que tiene el poder suficiente para imponer su imagen del orden y de la justicia: no cabe duda de que Shakespeare, en este sentido, está más cerca de Maquiavelo (o de Marx) que de Locke (o de Kant)" (Grüner, 2000. p. 146). 
Sandra Rocío Gamboa Rubiano

A propósito de una verdad contrahegemónica: aprendizajes desde la decisión de la Corte Penal Internacional...

\section{INTRODUCCIÓN}

A pesar de la tendencia a una verdad hegemónica, que obedece a lo que Foucault (1996a y 2012) denominó como un discurso de poder específico, las resistencias de las víctimas de graves violaciones de derechos humanos han sentado las bases de potenciales verdades contrahegemónicas. Una de las más interesantes implicaciones de este fenómeno es su posibilidad transformadora de la administración de justicia, tanto en el ámbito nacional como en los espacios de justicia internacional.

Esto implica reconocer, con Bordieu (2008), que en cuestiones de las representaciones sociales en la construcción de la verdad, la que se afirma como científica obedece a una particular fuerza social, que se encuentra en el poder. Formas de verdad que emergen del poder y que, como advirtiera Foucault (1996a), se definen, entre otros espacios, a partir de la práctica penal. La posibilidad de una verdad contrahegemónica (Gamboa, s.f.), a través de la cual las víctimas de los crímenes del poder buscan materializar sus derechos, reside en múltiples instancias y a través de diversas proyecciones. Todas ellas implicarían determinarse a superar los mitos, implicándolos de forma pedagógica y conectándolos con la construcción de una verdad histórica (Correas, 2006).

En este texto se aproximará a una de las posibilidades de construcción de la verdad contrahegemónica: los procesos penales internacionales. Por su papel central en la construcción contemporánea de un Derecho Penal Internacional (DPI), que respalde los derechos de las víctimas, se tratará la reciente decisión de la Corte Penal Internacional (CPI, ICC) revisando una actuación de la Fiscalía de la CPI (OFCPI, OTP). Se visita así la postura de esa Corte sobre el cierre del Examen Preliminar concerniente a la situación de las embarcaciones de la Unión de Comoras, la República Helénica y el Reino de Camboya. Allí se pronunció sobre los crímenes internacionales realizados en el ataque del 31 de mayo de 2010, del Estado de Israel, contra embarcaciones integrantes de "la Flotilla de la Libertad". Es una decisión que desafió los argumentos de cierre expuestos por la Fiscalía del tribunal penal internacional permanente, y reiteró su compromiso con la materialización de los derechos de las víctimas a través de la necesidad de un adecuado análisis de contexto. 


\section{Problema de Investigación}

¿La decisión de la Corte Penal Internacional sobre el cierre de este Examen Preliminar evidencia la trascendencia de escucha de la verdad contrahegemónica dentro de los procesos penales?

\section{Hipótesis de trabajo}

La decisión de la CPI al revisar el cierre del Examen Preliminar de la situación de las embarcaciones de la Unión de Comoras, la República Helénica y el Reino de Camboya reitera que es impostergable considerar la verdad de las víctimas dentro de los procesos penales internacionales. Esta verdad, en casos de crímenes internacionales a manos de agentes del Estado, se advierte como contrahegemónica, carácter que además determina su trascendencia.

\section{Estrategia Metodológica}

Este texto es un artículo de reflexión resultado de una investigación, a través del cual se adelanta un estudio teórico sobre las posibilidades de incidencia de una verdad contrahegemónica; esto es, de las víctimas de crímenes del poder, en el ejercicio del Derecho Penal.

A través del método analítico se contrasta la teoría foucoltiana sobre la verdad como discurso de poder específico, con la reciente decisión de la CPI relativa al ataque contra la "Flotilla de la Libertad", por el Estado de Israel. Se utiliza una estrategia de investigación exploratoria al discutir una categoría construida por la autora - la verdad contrahegemónica- y las posibilidades de las víctimas de los crímenes de Estado en la CPI. También se aplica una metodología descriptiva para asumir debates propios de las dinámicas actuales de la actuación de la CPI. 
Sandra Rocío Gamboa Rubiano

A propósito de una verdad contrahegemónica: aprendizajes desde la decisión de la Corte Penal Internacional...

\section{Resultados}

\section{Del derecho a la verdad hacia la configuración de una verdad contrahegemónica}

Como resalta Andreu-Guzmán (2012), el reconocimiento del derecho a la verdad de las víctimas es una conquista histórica que ha iniciado desde el Derecho Internacional Humanitario. Desde allí, ha transitado hasta la configuración de un corpus iuris internacional (Andreu-Guzmán, 2012) y a su concreción en escenarios como el latinoamericano, donde ha constituido un reclamo trascedente para superar regímenes represivos (Abregú, 2008). Esta constatación ha tenido particular incidencia en los tribunales de Derecho Internacional de los Derechos Humanos, no solo en razón de que un caso latinoamericano ayudó a configurar el primer reconocimiento del derecho a la verdad en el sistema de Naciones Unidas (Andreu-Guzmán, 2012, p. 23). Además, como destaca Rincón (2005): "La voz de la víctima, esto es, la víctima y su relato, adquieren entidad autónoma y autorizada en el espacio de los tribunales internacionales de los derechos humanos" (p. 339).

En el ámbito interamericano, este derecho se reconoció y desarrolló en vinculación con la responsabilidad internacional del Estado, en el aún actual fenómeno de la desaparición forzada de personas (Andreu Guzmán, 2012; CIDH, 2014). En dicho entorno, se reafirma la necesidad de develar la verdad histórica en conexión con la administración de justicia penal. Esto es, la complementación entre la verdad histórica y la judicial (CorteIDH, 2006, párr. 160; 2012, pár. 176). Ello explica algunas de las caracterizaciones de la verdad en el sistema interamericano: (i) como condición para evitar la repetición (CorteIDH, 2002, pár. 77; 2004, párr. 259; 2009, párr. 179); (ii) como principio, base de una democracia (CorteIDH, 1988, párr. 8; 2010, párr. 201), cuya aplicación conduce hacia la justicia (CorteIDH, 2004a, párr. 82); (iii) como derecho de las víctimas y sus familiares (CorteIDH, 1988, párr. 181; 2006a, párr. 165; 2006b, párr. 246; 2006c, párr. 197; 2006d, párr. 219; 2009a, párr. 117; 2009b, párr. 118; 2011, párr. 243; 2011a, párr. 173; 2010, párr. 201) y como derecho de la sociedad en su conjunto (CorteIDH, 1988, párr. 181; 2005a, párr. 117; 2007, párr. 195; 2010a, párr. 158; 2010b, párr. 234; 2011a, párr. 170). 
Asimismo, la vinculación entre la desaparición forzada como grave violación de derechos humanos y el reconocimiento del derecho a la verdad se reitera en la Resolución 2005/66. Este texto de la extinta Comisión de Derechos Humanos de Naciones Unidas constituye uno de los primeros documentos internacionales que afirma ese derecho de las víctimas.

El decisivo reconocimiento por los tribunales del Derecho Internacional de Derechos Humanos ha implicado críticas de quienes consideran que el Derecho debe encontrarse a espaldas de transformaciones sociales y políticas. En este último grupo, para autores como Malarino (2010) la jurisprudencia de la Corte Interamericana de Derechos Humanos (CorteIDH) se ha convertido, entre otros, en un escenario de activismo judicial. Esta afirmación advierte sobre la obtención de eventuales verdades contrahegemónicas a través del reconocimiento de la personalidad jurídica internacional de los individuos, como también de los obstáculos sobre los que se ha alzado la afirmación de este derecho. La aserción del derecho a la verdad de las víctimas de crímenes de Estado implica un enfrentamiento de la verdad oficial a través del Derecho, con pretensiones de transformación del futuro, particularmente mediante la búsqueda de la no repetición.

Pero, además, esta afirmación explica la contradicción que en principio podría expresar la necesidad de una verdad contrahegemónica de las víctimas en la Corte Penal Internacional. Esto es, habiéndose establecido que el reconocimiento generador del derecho a la verdad se enlaza con su titularidad en las víctimas, además bajo dimensiones individuales y colectivas. En dicho norte, el Derecho Internacional de los Derechos Humanos subraya la instancia de materialización de los propios derechos, y además destaca su vinculación con la no repetición (CorteIDH, 1988, párr. 181; 2002, párr. 91; 2003, párr. 274; 2004b, párr. 98; 2005, párr. 147; 2005a, párr. 63; 2010, párr. 201; 2012a, párr. 217; 2012, párr. 176). Entonces, ¿̨por qué se alude a la necesidad de una verdad contrahegemónica? ¿La verdad que puede recogerse en los tribunales internacionales de derechos humanos acaso no es suficiente? Interrogantes trascedentes, con mayor razón cuando se registra que la verdad histórica es un concepto que debe mostrarse contextualizado, y en el que es capital determinar la autoridad normativa de quien la establece, así como su significado a efectos de lo que 
Sandra Rocío Gamboa Rubiano A propósito de una verdad contrahegemónica: aprendizajes desde la decisión de la Corte Penal Internacional...

Rincón (2005) advierte como elaboración de la memoria y definición de la identidad de una sociedad.

Una primera afirmación de la necesidad de una verdad contrahegemónica es la tradicional divergencia entre los reconocimientos normativos de los derechos y su concreción en la realidad. Gallardo (2009), bajo una aproximación sociohistórica a los derechos humanos, advierte la vinculación de estos con la internalización de la cultura, sin la cual no es posible su eficacia. Además, la vigencia del reconocimiento del derecho de las víctimas a la verdad depende de nuevo del interés del poder por su refrendación. Ello cobra expresiones difíciles en relación con la criminalidad de Estado.

En un segundo orden, la verdad contrahegemónica coincide con el reconocimiento de que las víctimas acuden a los tribunales internacionales tras agotar las posibilidades de ejercicio de sus derechos en las órbitas nacionales. Ello no solo se explica a partir de la imposición de una tendencia de jurisdicción complementaria en los tribunales de derechos humanos, y en los de Derecho Penal Internacional que no se encuentran bajo el control del Consejo de Seguridad de Naciones Unidas (Gamboa, s.f.). También ha sido reconocido en el sistema de la Corte Penal Internacional, donde la fiscalía señala: "In the absence of the ICC launching an investigation, the prospects of holding those responsible to account in any court of law is essentially nil" (ICC-01/13, January 29, 2015, párr. 58).

Un tercer grupo de respuestas provendría de la verificación sociológica de la verdad como derecho de las víctimas. En otros trabajos se ha profundizado en la vinculación de la construcción de la verdad contrahegemónica con la llegada de una sociología de las ausencias (Gamboa, s.f.). Tales propósitos precisarían: (i) la superación de la razón instrumental mediante la memoria, al reconocer las estructuras de poder a cargo de los crímenes de Estado; (ii) admitir la existencia de una razón de Estado que sigue protegiendo la criminalidad del poder, a pesar de su superación normativa a partir del Derecho de Núremberg; (iii) la exigencia de declarar la verdad de las víctimas y que ello, además, tenga efectos sociales, políticos, jurídicos y culturales; y (iv) el reconocimiento de los sujetos individuales y colectivos como realizadores de la idea de humanidad (Gamboa, s.f.). En este orden: 
"La verdad contrahegemónica para las víctimas de crímenes de Estado busca una revalorización de la pretensión de humanidad, cercana a la que acompañó la eclosión de los derechos humanos de la segunda posguerra, pero transformando la apariencia ética del Estado liberal por una realidad ética del Estado. Por lo pronto, exige la transformación de la unívoca pretensión del Derecho como herramienta de regulación. De ahí que se observe una correspondencia directa entre la construcción jurídica del derecho a la verdad y la limitación de la razón de Estado" (Gamboa, s.f.).

Entonces, desde una perspectiva sociológica, la verdad contrahegemónica puede señalar las condiciones para la expresión de la dimensión colectiva de este derecho. Con ello, de nuevo, refuerza la garantía de no repetición. Ahora, es necesario observar cómo pudiera tener ello ocurrencia en el contexto del Examen Preliminar en la CPI.

\section{El Examen Preliminar como contexto de ejercicio para la verdad contrahegemónica}

El Examen Preliminar se concibe en el procedimiento ante la Corte Penal Internacional como un conjunto de actividades que se desarrollan ante la Fiscalía y que pueden ser objeto de pronunciamiento de las Salas de Cuestiones Preliminares respectivas. Estas actividades buscan determinar: los elementos de competencia como base razonable, la admisibilidad y el Interés de la Justicia; y, a su vez, implican algunas rutas de actuación que pueden sintetizarse en un ejercicio serio de investigación.

En relación con el primer aspecto, a través del Examen Preliminar se busca determinar:

a) La competencia como base razonable. En este extremo, se verifica si existe una base razonable para considerar la existencia de crímenes internacionales de competencia de la CPI, y, por tanto, bajo un estándar de prueba mínimo de conformidad con las exigencias del Estatuto de Roma (ER), las Reglas de Procedimiento y Prueba (RPP), la jurisprudencia de la corte y los documentos de política de la Oficina de la Fiscalía de la CPI (OFCPI, OTP) [ER, Artículo 15 (1)-(3), 15(6) y 53 (1) a)-c). RPP, Reglas 48 y 104. ICC-02/11-14, par. 
Sandra Rocío Gamboa Rubiano A propósito de una verdad contrahegemónica: aprendizajes desde la decisión de la Corte Penal Internacional...

24; ICC-01/09-19, par. 27 y 35; ICC-OTP-02/11-3, par. 37. OTP, 2013, par. 34].

En este panorama no se precisa que la base razonable sea concluyente, pues incluso frente a la ausencia de claridad debería avanzarse hacia una investigación (ICC01/13, July 16, 2015, par. 13). Similar decisión debe tener lugar de evidenciarse inferencia razonable de la existencia de por lo menos un solo crimen internacional de competencia de la CPI: "the Prosecutor shall open an investigation, as only by investigating could doubts be overcome" (ICC-01/13, July 16, 2015, par. 13).

b) La admisibilidad en referencia con el sistema de la CPI. En sede de la admisibilidad, se examina si se cumplen los elementos de complementariedad y gravedad necesarios para el ingreso de la situación y sus casos en el sistema de la Corte Penal Internacional (ER, Art. 17).

En relación con la complementariedad, se puede verificar que existe falta de voluntad para adelantar auténticos procedimientos nacionales (ICC-01/04-01/07-1497, September 25, 2009, par. 78; OTP, 2013, par. 47), o concluir que estos no son posibles dado el colapso total o sustancial del sistema o, en razón de la imposibilidad del Estado para acopiar pruebas, hacer comparecer al acusado o adelantar el juicio. Este análisis de complementariedad debe realizarse sobre las condiciones existentes al momento de la indagación de los hechos (ICC-02/04-01/05-377, March 10, 2009, par. 49-52; OTP, 2013, par. 47), sobre los potenciales casos identificados (OTP, 2013, par. 46. ICC-02/11-01/11, September 25, 2009, par. 78) y los eventuales más altos responsables comprometidos (ICC-01/09-01/11-307, August 30, 2011, par. 1, 47; ICC-01/09-02/11-274, August 30, 2011, par. 1 \& 46; OTP, 2013, par. 47).

La CPI ha señalado que la gravedad debe evidenciarse a través del análisis de potenciales casos (ICC-01/09-19, March 31, 2010, par. 50 \& 188. ICC-02/11-14, October 3, 2011, par. 202-204), y bajo el contexto de interacción entre el contexto y el específico modus operandi de los crímenes verificados (ICC-01/09-19, March 31, 2010, par. 61. ICC-02/11-14, October 3, 2011, par. 17). La jurisprudencia de la ICC también resalta que dentro de la gravedad deben evaluarse los eventuales efectos de una decisión de no proceder a la investigación, con lo que reitera la importancia 
de la intervención de la CPI en general y del adelantamiento del Examen Preliminar en particular, como mecanismo disuasorio de la configuración de nuevos crímenes (ICC-01/13, January 29, 2015, par. 25). Asimismo, como sucede en el análisis de complementariedad general, las indagaciones deben comprender a quienes puedan constituir los más altos responsables (ICC-01/09-19, March 31, 2010, par. 50; OTP, 2013, par. 42 y 72; Regulations of the Office of the Prosecutor, Regulation 34; ICCBD/05-01-09, April 23, 2009. ICC-01/09, November 26, 2009, par. $55 \& 78$ ).

c) La verificación del Interés de la justicia. Bajo esta lógica se verifica si el procesamiento de la situación es acorde con el Interés de la Justicia, comprendiendo la explícita consideración de la gravedad del crimen, los intereses de las víctimas y las circunstancias particulares de los acusados (ER, art. 53.1.c) y 53.2.c); OTP, 2007, par. 5 y 7).

Desde el Estatuto de Roma, las Reglas de Procedimiento y Prueba, las decisiones de la CPI y los documentos de política de sus órganos, entre otros, se desprenden dos obligaciones centrales para la Fiscalía en relación con la forma como debe adelantar las investigaciones dentro del Examen Preliminar:

En primer lugar, es titular de deberes de investigación seria, como se desprende del Artículo 21 del ER, pues en virtud del derecho aplicable deben aplicarse los derechos humanos internacionalmente reconocidos. A partir de allí, y en conjunción con el artículo 54.1 del ER, la Fiscalía de la CPI puede solicitar información a diversas fuentes confiables de origen estatal, internacional y no gubernamental (OTP, 2013). Finalmente, se debe permitir que las víctimas puedan ser escuchadas en todas las etapas de las actuaciones (ICC-ASP/11/38, November 5, 2012). En un segundo orden, la OFCPI debe hacer una aproximación estructural al contexto en que han ocurrido los potenciales crímenes internacionales, por lo que no le es posible segmentarlo (ICC-01/11-01/11-1, par. 26; ICC-02/05-01/09-3, March 4, 2009, par. 166-169; ICC-02/05-157-AnxA, July 14, 2008, par. 86-87, 349-354, 356). Sobre este punto, trascendente en la decisión de la CPI sobre el cierre del Examen Preliminar atinente a la "Flotilla de la libertad" en Gaza, se retornará en el siguiente acápite. 
Sandra Rocío Gamboa Rubiano A propósito de una verdad contrahegemónica: aprendizajes desde la decisión de la Corte Penal Internacional...

Por ahora, corresponde observar las eventuales vinculaciones que emergen del ejercicio del derecho a la verdad en el contexto del Examen Preliminar en el sistema de la CPI. Debe reconocerse que la admisión de la verdad como derecho de las víctimas también ha dejado impronta dentro del sistema de la Corte Penal Internacional. Ello obedece principalmente a la construcción jurisprudencial de la Corte, y, desde luego, a la particular influencia del movimiento de derechos humanos en la edificación del Estatuto de Roma. Se refleja, a su vez, por ejemplo, en el concepto de víctima incorporado en el ER a partir de la Declaración de Naciones Unidas sobre los principios fundamentales de justicia para las víctimas de delitos y del abuso de poder y, por otro lado, los Principios y directrices básicos de Naciones Unidas sobre el derecho de las victimas de violaciones manifiestas de las normas internacionales de derechos humanos y de violaciones graves del derecho internacional humanitario.

La incidencia de estas declaraciones se reafirma en la actualidad dentro del sistema, a través de la jurisprudencia de la CPI, y en documentos como la Estrategia en relación con las víctimas de la ICC y la revisión de la Estrategia (ICC-ASP/8/45, November 10, 2009, par. 6; ICC-ASP/11/38, November 5, 2012). Además, como se observó, el artículo 21 del ER reconoce expresamente la trascendencia del Derecho Internacional de los Derechos Humanos, al aludir a los derechos humanos internacionalmente reconocidos. Desde luego, el derecho a la verdad constituye un derecho humano internacionalmente reconocido, cuya aplicación en el contexto de la CPI se desprende del artículo 21 (3) del Estatuto de Roma. Además, la jurisprudencia de la CPI registra el derecho de las víctimas a la verdad (ICC, PTC-I, 2006; ICC, PTC-I, 2008).

Este reconocimiento se debe leer en el contexto de las luchas políticas contra su materialización, así como en el ámbito de las restricciones impuestas al ejercicio de los derechos de las víctimas en el Examen Preliminar desde el Estatuto, mientras se fortalecían las posibilidades en cabeza de la Fiscalía de la Corte Penal Internacional.

En la primera vía, como sucede con las críticas por el reconocimiento de los derechos de las víctimas en el sistema interamericano de protección; en el contexto académico relativo al Derecho Penal Internacional, algunas voces rechazan la afirmación del Derecho a la verdad. Entre ellas, MacGonigle Leyh (2013) considera que este derecho es un concepto indeterminado del Derecho Internacional de los Derechos 
Humanos, y llama a la CPI a limitar su aplicación. Estas afirmaciones emergen de su visión restrictiva sobre la necesidad del descubrimiento de la verdad, pues restringe el derecho a la verdad a la pretensión de que exponerse a ella podría aliviar el sufrimiento de las víctimas. Los acápites anteriores de este artículo han desvirtuado dicho argumento.

Entre las situaciones de restricción a las posibilidades de intervención de las víctimas en la CPI, debe mencionarse la limitación para el ejercicio de la revisión oficiosa por parte de la Corte, en relación con una decisión de cierre de Examen Preliminar, en la que se defina no proceder a la investigación. En principio, solo procedería esta revisión si la decisión de la Fiscalía se ha sostenido únicamente con fundamento en los párrafos 1(c) o 2(c) del Artículo 53(3)(b) del ER. Además, en la búsqueda de la revisión de fondo de la CPI sobre una decisión de esta naturaleza, el ejercicio de la impugnación en relación con las restantes circunstancias estaría reservado a las partes dentro de los procedimientos, lo que en principio impediría dicho ejercicio a las víctimas (ER, art. 82(1)(a)).

No obstante ello, considerando que sería factible formular la existencia de principio de primacía de lo material sobre lo formal, precisamente con sustento en los derechos humanos internacionalmente reconocidos, la conclusión puede ser diferente. Con esto se hace referencia a la hipótesis en la que la Fiscalía de la Corte Penal Internacional pueda afirmar que procede al cierre con fundamento en criterios propios del análisis de competencia, cuando en realidad lo hace a partir del Interés de la Justicia.

\section{La decisión de la Corte Penal Internacional sobre el cierre del Examen Preliminar en la situación de las embarcaciones de la Unión de Comoras, la República Helénica y el Reino de Camboya}

Facilitar la intervención de las víctimas de crímenes de Estado o de las víctimas del poder en los momentos previos a la autorización de una investigación constituye uno de los momentos más sensibles para que ingrese una intervención hegemónica (Gamboa Rubiano, s.f.). Esta conclusión parece empezar a corroborarse a través de la decisión de la CPI sobre el cierre del Examen Preliminar frente al asunto 
Sandra Rocío Gamboa Rubiano A propósito de una verdad contrahegemónica: aprendizajes desde la decisión de la Corte Penal Internacional...

de la "Flotilla de la Libertad" en Gaza. Justamente, la necesidad de una verdad contrahegemónica en el sistema de la Corte Penal Internacional se ratifica con algunas de las consideraciones expuestas por la CPI en relación con el cierre relativo a la "Flotilla". Ello obliga a un breve recuento de dicha situación en este sistema.

Se recordará que, desde el 3 de enero de 2009, Israel impuso un bloqueo naval sobre las costas que rodean 20 millas náuticas desde la costa de la Franja de Gaza (OTP, 2014, párr. 10). En desafío, desde el movimiento social internacional de los derechos humanos, se configuró el Movimiento Gaza Libre (OTP, 2014, párr. 11). El 31 de mayo de 2010, una Flotilla de ayuda humanitaria denominada "Flotilla de la Libertad", se dirigía a Gaza, conformada por ocho barcos con más de 700 pasajeros correspondientes a unos 40 países (OTP, 2014, párr. 11). La OFCPI reconoce que la flotilla buscaba prestar ayuda humanitaria a Gaza, romper el bloqueo israelí y llamar la atención internacional sobre la situación en estos territorios (OTP, 2014, párr. 11).

Seis embarcaciones de la flotilla fueron atacadas el 31 de mayo de 2010 por agentes de las Fuerzas de Defensas Israelíes (OTP, 2014, párr. 2). Una séptima embarcación se había retirado por dificultades mecánicas y otra se retrasó al momento de la salida, y fue interceptada posteriormente (UN, 2010). En la embarcación Mavi Marmara, de bandera de la Unión de Las Comoras, fueron asesinadas nueve personas, y una más falleció meses después a consecuencia de las heridas allí recibidas. Nueve de estos ciudadanos eran de nacionalidad turca, y uno más tenía doble nacional, incluyendo la estadounidense (OTP, 2014, pár. 13).

Sobre estos hechos se abrieron investigaciones en diversos contextos relativos a la "justiciabilidad" de los derechos humanos, que ponen de presente la profusión de discursos de verdad, sustentados en las fuerzas sociales que los representan. En noviembre de 2012, el Fiscal ante la Audiencia Nacional de Espańa encontró que el ataque constituía un crimen de lesa humanidad (Reino de España, 2012), pero el caso fue cerrado en razón a las Modificaciones de la Ley Orgánica del Poder Judicial (Reino de España, 2015). La Asamblea General de Naciones Unidas publicó en septiembre de 2010 un informe de su misión de investigación internacional, establecida por el Consejo de Derechos Humanos en la resolución 14/1 del 2 de 
junio de 2010 (UN, 2010). Según esta Misión, los hechos incluían graves violaciones de derechos humanos e infracciones al Derecho Internacional Humanitario. Por otra parte, en septiembre de 2011, se publicó el "Informe Palmer" de la Comisión de Investigación del Secretario General sobre los mismos hechos, que concluyó que el bloqueo contra Gaza era legal, y que el ataque contra el Mavi Marmara era justificado pero presentaba un uso excesivo de la fuerza (UN, 2011).

La situación de las embarcaciones fue remitida a la CPI por las autoridades de la Unión de Comoras, el 14 de mayo de 2013. El mismo día, el entonces Fiscal de la CPI anunció la apertura del Examen Preliminar (OTP, 2014, par. 2, 5 y 7). El 19 de mayo siguiente, la Presidencia de la CPI asignó su conocimiento a la Sala de Cuestiones Preliminares I (OTP, 2014, párr. 7). La Unión de Comoras aclaró dentro del procedimiento ante la OFCPI que el ámbito territorial de la remisión implicaba a la embarcación comorense "Mavi Marmara", así como a los demás buques de la "Flotilla de la Libertad", registrados en Estados partes de la CPI (OTP, 2014, par. 6), como el "Rachel Corrie" de Camboya y el "Eleftheri Mesogios/Sofia" de Grecia (OTP, 2014a).

En su informe sobre el artículo 53.1, en noviembre de 2014, la OFCPI concluyó la existencia de una base razonable para creer la existencia de crímenes internacionales de competencia de la Corte, en particular crímenes de guerra a bordo del "Mavi Marmara". Pero, al tiempo, señaló que no se evidenciaban los elementos propios de la gravedad para poder proceder a una investigación, por lo que decidió cerrar el Examen Preliminar (OTP, 2014, párr. 3).

En respuesta a ello, los representantes del Gobierno de Las Comoras pidieron la revisión de la decisión de archivo de la Fiscalía, a fin de que la CPI solicitara a la OFCPI su reconsideración (ICC-01/13, January 29, 2015). Esta acción se enmarca en una intensa actividad legal de exigencia de pronunciamiento ante la OFCPI por parte de la Unión de Comoras. Dichas actuaciones revirtieron en el informe de cierre de examen preliminar, y quizá marcan la diferencia que la acción judicial decidida ante la OFCPI puede ofrecer en términos de ejercicio de los derechos, frente a otras situaciones como las de Colombia, que permanece hace largos años ante la CPI. También contrasta con la decisión de cierre del Examen Preliminar de Honduras, en 
la que la OFCPI no dio respuesta a un documento de argumentación jurídica de las víctimas hondureñas, como fue denunciado en octubre de 2015 (Hondurastierralibre. com, 2015).

Asimismo, las situaciones de las embarcaciones de Las Comoras, y de Honduras, ponen de manifiesto la necesidad de reconocimiento del derecho de las víctimas para acudir ante la CPI, en los casos donde la Fiscalía dice cerrar el Examen Preliminar con fundamento en elementos diferentes al Interés de la Justicia. Mientras que, en las decisiones basadas de manera exclusiva en el Interés de la Justicia, la CPI puede adelantar una revisión oficiosa; en los restantes casos, en principio, solo podría intervenir el Estado que ha remitido la situación ante la CPI.

En la situación relativa a la "Flotilla de La Libertad", la Sala de Cuestiones Preliminares de la CPI se pronunció el 16 de julio de 2015, en una decisión que se considera trascendental para la reflexión propuesta en este artículo, por la necesidad de revisar adecuadamente el contexto (ICC-01/13, July 16, 2015). Ello, desde luego, tiene que ver con la necesidad de incorporar un examen acucioso de las diversas formas de verdad, para tomar una decisión apropiada.

Repárense en que la existencia de informes en el contexto de la Organización de Naciones Unidas, con resultados divergentes (Misión Internacional y el Informe Palmer) da lugar al reconocimiento de puntos de vista diferentes sobre un mismo aspecto. Además, ello tiene lugar en el contexto de un conflicto en el que el Estado de Israel ha sido denunciado en oportunidades anteriores, y en el cual la respuesta del Derecho Penal Internacional había sido reducida. Una muestra de esta afirmación es el desistimiento de competencia en razón a que Palestina, con anterioridad al año 2012, no se consideraba Estado integrante de la Organización de Naciones Unidas. Solo hasta el 29 de noviembre de 2012 Palestina empezó a ser considerado como Estado No Miembro Observador, a través de la resolución A/67/19.

Pero los retos siguen, a pesar de este mínimo reconocimiento, y de que se adelanta otro Examen Preliminar concerniente a la situación de Palestina, particularmente por los hechos ocurridos en el territorio ocupado por Israel desde el 13 de junio de 2014. La existencia de esta situación fue una de las razones alegadas por la OFCPI 
para cerrar el Examen Preliminar sobre las embarcaciones de Las Comoras, Grecia y Camboya. No obstante, el cierre del Examen Preliminar en este último caso dio oportunidad a la CPI para pronunciarse sobre las actividades desarrolladas por la misma Corte bajo un Examen Preliminar. Así, la CPI destaca en lo que corresponde al análisis integral del contexto que, según el artículo 53.1 del ER, la OFCPI debía evaluar adecuadamente los hechos pertinentes. A partir de esta afirmación, se resalta la pertinencia de una verdad contrahegemónica, donde las voces silenciadas llegaron esta vez de mano del pequeño país de Las Comoras, un conjunto de tres islas en el sureste africano. Y lo hicieron de manera determinante ante la instancia judicial, después de haber sido desoídos en el contexto de la Fiscalía de la CPI.

En efecto, la CPI subraya que, a fin de sustentar la decisión de cierre del Examen Preliminar sobre la situación de la "Flotilla de la Libertad", la OFCPI ignoró, en contravía de lo que había sido solicitado por Las Comoras al remitir la situación ante la CPI, que, si bien se habían presentado diez homicidios, ello además tenía en el contexto del conflicto de Israel contra Palestina (ICC-01/13, January 29, 2015, par. 25). La CPI recalca que la omisión del análisis integral del contexto de las situaciones puede llevar a omitir la existencia de crímenes de competencia de la ICC (ICC01/13, July 16, 2015, par. 17 \& 18).

Sobre la necesidad de una seria revisión contextual, también se había pronunciado la misión de observación de Naciones Unidas, lo que le permitió concluir la existencia de una crisis humanitaria en Gaza al momento de los hechos, un bloqueo ilegal y una acción contra la "Flotilla de la Libertad", igualmente ilegal (UN, 2010). Así, evidenció que Israel realiza un castigo colectivo contra la población civil de Gaza, absolutamente ilegal ante el Derecho Internacional (UN, 2010), donde el ataque contra la flotilla evidenciaba: "an unacceptable level of brutality" (UN, 2010, par. 264).

No podría ser menos cuando la completa revisión de la situación y su contexto permite infirmar o descartar, por ejemplo, la existencia de un ataque como línea de conducta. En el caso de crímenes de lesa humanidad, y siguiendo los contornos del Artículo 7(2)(a) del ER, un ataque se comprende como una línea de conducta que involucra una serie global de acontecimientos que demuestran un cierto patrón 
de conducta. Además, dicho ataque debe involucrar cualquier forma de violencia contra la población civil, sin que ello implique afirmar que pueda tratarse de un simple agregado de actos aleatorios (ICC-01/13, July 16, 2015. ICC-02/11-01/11656-Red, June 12, 2014, par. 209-212 ; ICC-01/04-01/07-3436-tENG, March 7, 2014, par. 1101; ICC-02/11-14, October 3, 2011, par. 31; ICC-01/09-19, March 31, 2010, par. 80; ICC-01/05-01/08-424, June 15, 2009, par. 75).

\section{Conclusiones: Aprendizajes para el Derecho Nacional}

Las víctimas concurren a los tribunales internacionales en ejercicio de resistencia mediante el Derecho, y con la determinación de su verdad contrahegemónica ponen de presente junto al pensamiento crítico (Foucault, 1987 y 1996b; Santos, 2007) que la verdad impuesta desde el poder puede y debe ser descubierta.

Si bien estos tribunales, como sucede en el Derecho nacional, están eventualmente mediados por condicionamientos referidos a la verdad que expresan, pueden constituir una herramienta de racionalización de la administración de justicia internacional. Una administración de justicia, que como revelan situaciones ante la CPI como las de Colombia y Honduras, entre otras, pueden depender más de estrategias diplomáticas que de aplicación del derecho. Por ello, resulta trascendente que arriben hacia las instancias judiciales y no se determinen únicamente en los espacios de incidencia política a cargo de la Fiscalía.

En esas condiciones, la verdad contrahegemónica permite ingresar en la construcción contrahegemónica de la memoria (Gamboa, s.f.). Con ello, podría postular posibilidades reales de relacionamiento entre la verdad histórica y la verdad jurídica. Ello, por supuesto, no podría tener lugar sin permitir la participación de las víctimas, incluso en los procesos de construcción de la prueba dentro del proceso penal. Se hace realmente impostergable en los hechos que podrían calificarse inicialmente como graves violaciones de los derechos humanos y que pueden constituir crímenes internacionales bajo el Estatuto de Roma de la Corte Penal Internacional. 


\section{REFERENCIAS}

Abregú, M. (2008). Derechos humanos para todos: de la lucha contra el autoritarismo a la construcción de una democracia inclusiva, una mirada desde la Región Andina y el Cono Sur. Revista Sur, (8), 6-41.

Andreu-Guzmán, F. (2012). Derecho a la verdad y derecho internacional. Bogotá: Comisión Colombiana de Juristas.

Bordieu, P. (2008). Homo Academicus. Buenos Aires: Siglo XXI Editores.

Comisión Interamericana de Derechos Humanos -CIDH- (2014). Derecho a la verdad en América. OEA/Ser.L/V/II.152

Correas, O. (2006). Los derechos humanos. Entre la historia y el mito. Revista Critica Juridica, (25), 269-292.

Foucault, M. (1996a). La verdad y las formas juridicas. En E. Lynch (Trad.). Barcelona: Gedisa.

Foucault, M. (1996b). Genealogía del racismo. En T.A. Tzveibel (Trad.). La Plata: Editorial Altamira.

Foucault, M. (1987). La casa de la locura. En F. Basaglia y F. Basaglia Ongaro. (Eds.) Los crímenes de la paz. Investigación sobre los intelectuales y los técnicos como servidores de la opresión (pp. 135-150) (3 Ed.). México: Siglo XXI Editores.

Foucault, M. (2012). Vigilar y Castigar. Nacimiento de la Prisión (2a Ed., 3a Reimp). Buenos Aires: Siglo XXI Editores.

Gallardo, H. (2009). Derechos Humanos como Movimiento Social (2a Reimp.). Bogotá: Departamento Ecuménico de Investigación-Ediciones Desde Abajo. 
Gamboa-Rubiano, S. Solicitudes de las victimas de crimenes de Estado ante la Corte Penal Internacional: Su incidencia en la apertura de una investigación. (Tesis doctoral inédita). Universidad Santo Tomás. Colombia.

Grüner, E. (2000). El Estado: pasión de multitudes. Spinoza versus Hobbes, entre Hamlet y Edipo. En Atilio Borón (comp.). La filosofía politica moderna. De Hobbes a Marx (pp. 143-165). Buenos Aires: Clacso.

Hondurastierralibre.com (2015). Honduras Tierra Libre: Organizaciones de Derechos Humanos de Honduras impugnaran Resolución de la Fiscalia de la CPI. Recuperado de: http:// www.hondurastierralibre.com/2015/10/organizaciones-de-derechos-humanos-de.html. [Accedido: 21 enero 2016].

Malarino, E. (2010). Activismo judicial, punitivización y nacionalización: Tendencias antidemocráticas y antiliberales de la Corte Interamericana de Derechos Humanos. En Grupo Latinoamericano de Estudios sobre Derecho Penal Internacional. Sistema Interamericano de Protección de los Derechos Humanos y Derecho Penal Internacional (pp. 379425). Montevideo: Georg-August-Universität-Göttingen, Konrad Adenauer Stiftung.

MacGonigle Leyh, B. (2013). The Right to truth in International Criminal Proceedings: An indeterminate concept from Human Rights Law. In Yves Haeck, Brianne McGonigle Leyh, Clara Burbano-Herrera \& Diana Contreras-Garduno (Eds.). The Realisation of Human Rights: When Theory Meets Practice (pp. 293-312). Antwerp: Intersentia.

Reino de España (2012). Fiscalía de la Audiencia Nacional, Diligencias previas No. 197/2010, 16 de noviembre de 2012.

Reino de España (2015). Juzgado Central de Instrucción No. 5 de Madrid, Diligencias previas proceso abreviado No. 197/2010J, 10 de junio de 2015.

Rincón Covelli, T. (2005). La verdad histórica: una verdad que se establece y legitima desde el punto de vista de las víctimas. Estudios Socio-Jurídicos, 7, 331-354. 
Santos, B. De S. (2007). Beyond abyssal thinking: From global lines to ecologies of knowledges. I. F. Braudel Center (Review) (pp. 45-89).

United Nations, General Assembly (2014). Resolution adopted by the General Assembly on 29 November 2012, 67/19. Status of Palestine in the United Nations, A/RES/67/19

United Nations, Human Rights Council (27 September 2010). Report of the international fact-finding mission to investigate violations of international law, including international humanitarian and human rights law, resulting from the Israeli attacks on the flotilla of ships carrying humanitarian assistance, A/HRC/15/21.

United Nations (2011). Report of the Secretary-General's Panel of Inquiry on the 31 May 2010 Flotilla Incident, September 2011. Recuperado de: http://www.un.org/News/dh/ infocus/middle_east/Gaza_Flotilla_Panel_Report.pdf. [Accedido: 23 de enero de 2016].

\section{Decisiones de la Corte Interamericana de Derechos Humanos}

Corte Interamericana de Derechos Humanos -CorteIDH- (1988). Sentencia del 29 de julio de 1988, Serie C No. 4. Caso Velásquez Rodríguez vs. Honduras.

CorteIDH (2002). Sentencia del 22 de febrero de 2002, Serie C No. 91. Caso Bámaca Velásquez vs. Guatemala, Fondo.

CorteIDH (2003). Sentencia del 25 de noviembre de 2003, Serie C. No. 101. Caso Myrna Mack Chang vs. Guatemala, Fondo, Reparaciones y Costas.

CorteIDH (2004). Sentencia del 5 de julio de 2004, Serie C No. 109. Caso 19 comerciantes vs. Colombia, Fondo, Reparaciones y Costas.

CorteIDH (2004a). Sentencia del 25 de noviembre de 2004, Serie C No. 162. Caso Lori Berenson Mejía vs. Perú, Fondo, Reparaciones y Costas.

CorteIDH (2004b). Sentencia del 19 de noviembre de 2004, Serie C No. 116. Caso de la Masacre de Plan de Sánchez vs. Guatemala, Reparaciones. 
CorteIDH (2005). Sentencia del 15 de junio de 2005, Serie C No. 124. Caso de la Comunidad Moiwana vs. Suriname.

CorteIDH (2005a). Sentencia del 1 de marzo de 2005, Serie C No. 120. Caso de las Hermanas Serrano Cruz vs. El Salvador.

CorteIDH (2006). Sentencia del 26 de septiembre de 2006. Serie C No. 154. Caso Almonacid Arellano y otros vs. Chile, Excepciones preliminares, fondo, reparaciones y costas.

CorteIDH (2006a). Sentencia del 22 de septiembre de 2006, Serie C No. 153. Caso Goiburú y otros vs. Paraguay.

CorteIDH (2006b). Sentencia del 4 de julio de 2006, Serie C No. 149. Caso Ximenes Lopes vs. Brasil.

CorteIDH (2006c). Sentencia del 6 de abril de 2006, Serie C No. 147. Caso Baldeón García vs. Perú.

CorteIDH (2006d). Sentencia del 31 de enero de 2006, Serie C No. 140. Caso de la Masacre de Pueblo Bello vs. Colombia.

CorteIDH (2007). Sentencia del 11 de mayo de 2007. Serie C No. 163. Caso de la Masacre de La Rochela vs. Colombia. Fondo, Reparaciones y Costas.

CorteIDH (2009). Sentencia del 23 de noviembre de 2009, Serie C No. 209. Caso Radilla Pacheco vs. Estados Unidos Mexicanos.

CorteIDH (2009a). Sentencia del 3 de abril de 2009, Serie C No. 196. Caso Kawas Fernández vs. Honduras, Fondo, Reparaciones y Costas.

CorteIDH (2009b). Sentencia del 22 de septiembre de 2009, Serie C No. 202. Caso Anzualdo Castro vs. Perú, Excepción Preliminar, Fondo, Reparaciones y Costas. 
CorteIDH (2010). Sentencia del 24 de noviembre de 2010, Excepciones preliminares, fondo, reparaciones y costas, Serie C No. 219. Caso Gomes Lund ("Guerrilla Do Araguaia”) vs. Brasil.

CorteIDH (2010a). Sentencia del 1 de septiembre de 2010, Serie C No. 217. Caso Ibsen Cárdenas e Ibsen Peña vs. Bolivia, Fondo, Reparaciones y Costas.

CorteIDH (2010b). Sentencia del 25 de mayo de 2010, Serie C No. 212. Caso Chitay Nechy otros vs. Guatemala, Excepciones Preliminares, Fondo, Reparaciones y Costas.

CorteIDH (2011) . Sentencia del 24 de febrero de 2011. Caso Gelman vs. Uruguay, Fondo y Reparaciones.

CorteIDH (2011a). Sentencia del 31 de agosto de 2011, Serie C No. 232. Caso Contreras y otros vs. El Salvador, Fondo, Reparaciones y Costas.

CorteIDH (2012). Sentencia del 29 noviembre de 2012, Serie C No. 258. Caso García y Familiares vs. Guatemala, Fondo Reparaciones y Costas.

CorteIDH (2012a). Sentencia del 4 de septiembre de 2012, Serie C No. 250. Caso Masacres Río Negro vs. Guatemala, Excepción Preliminar, Fondo, Reparaciones y Costas.

\section{Decisiones y documentos dentro del sistema de la Corte Penal Internacional}

International Criminal Court -ICC- (2009) Regulations of the Office of the Prosecutor. ICC-BD/05-01-09.

ICC (17 January 2006). ICC-01/04. Situation in the Democratic Republic of the Congo, Decision on the applications for participations in the proceedings of VPRS 1, VPRS 2, VPRS 3, VPRS 4, VPRS and VPRS 6.

ICC (2009). ICC-01/04-01/07-1497. Situation in the Democratic Republic of the Congo, Judgment on the Appeal of Mr. Germain Katanga against the Oral Decision of Trial Chamber II of 12 June 2009 on the Admissibility of the Case. 
ICC (13 May 2008). ICC-01/04-01-07. Situation in the Democratic Republic of the Congo, Decision on the Set of Procedural Rights Attached to Procedural Status of Victim at the Pre-Trial Stage of the Case.

ICC (14 July 2008). ICC-02/05-157-AnxA. Situation in Darfur, Sudan, Public Redacted Version of the Prosecutor's Application under Article 58.

ICC (4 March 2009). Pre-Trial Chamber I, ICC-02/05-01/09-3. Situation in Darfur, Sudan in the case of The Prosecutor v. Omar Hassan Ahmad Al Bashir ("Omar Al Bashir"), Decision on the Prosecution's Application for a Warrant of Arrest against Omar Hassan Ahmad Al Bashir.

ICC (10 March 2009). ICC-02/04-01/05. Situation in Uganda in the case of The Prosecutor $v$. Joseph Kony, Vincent Otti, Okot Odhiambo, Dominic Ongwen. Decision on the admissibility of the case under article 19(1) of the Statute.

ICC (15 June 2009). Pre- Trial Chamber II, ICC-01/05-01/08-424. Situation in the Central African Republic in the case of the Prosecutor $v$. Bemba, Decision Pursuant to Article 61(7)(a) and (b) of the Rome Statute on the Charges of the Prosecutor Against Jean-Pierre Bemba Gombo.

ICC, The Appeals Chamber (25 September 2009). ICC-02/11-01/11. Situation in the Republic of Cote D'ivoire in the case of the prosecutor v. Laurent Gbagbo, Judgment on the Appeal of Mr. Germain Katanga against the Oral Decision of Trial Chamber II of 12 June 2009 on the Admissibility of the Case.

ICC, Assembly of States Parties (10 November 2009). ICC-ASP/8/45. Report of the Court on the strategy in relation to victims.

ICC (26 November 2009). ICC-01/09. Situation in the Republic of Kenya, Request for authorization of an investigation pursuant to article 15. 
ICC (31 March 2010). Pre- Trial Chamber II, ICC-01/09-19. Situation in the Republic of Kenya, Decision Pursuant to Article 15 of the Rome Statute on the Authorization of an Investigation into the Situation in the Republic of Kenya.

ICC (23 June 2011). Pre- Trial Chamber III, ICC-OTP-02/11-3. Situation in the Republic of Côte d'Ivoire, Request for authorisation of an investigation pursuant to article 15.

ICC (27 June 2011). ICC-01/11-01/11. Situation in Libya. The Prosecutor v. Saif Al-Islam Gaddafi, Warrant of Arrest.

ICC (30 August 2011). ICC-01/09-02/11-274. The Prosecutor v. Francis Kirimi Muthaura, Uhuru Muigai Kenyatta y Mohammed Hussein Ali, Judgment on the appeal of the Republic of Kenya against the decision of Pre-Trial Chamber II of 30 May 2011 entitled "Decision on the Application by the Government of Kenya Challenging the Admissibility of the Case Pursuant to Article 19(2)(b) of the Statute.

ICC (30 August 2011). ICC-01/09-01/11-307. The Prosecutor v. William Samoei Ruto, Henry Kiprono Kosgey y Joshua Arap Sang, Judgment on the appeal of the Republic of Kenya against the decision of Pre-Trial Chamber II of 30 May 2011 entitled "Decision on the Application by the Government of Kenya Challenging the Admissibility of the Case Pursuant to Article 19(2)(b) of the Statute.

ICC (3 October 2011). Pre- Trial Chamber III, ICC-02/11-14. Situation in the Republic of Côte D'Ivoire. Decision Pursuant to Article 15 of the Rome Statute on the Authorization of an Investigation into the Situation in the Republic of Côte d'Ivoire.

ICC, Assembly of States Parties (5 November 2012). ICC-ASP/11/38. Court's Revised strategy in relation to victims.

ICC, Trial Chamber II (7 March 2014). ICC-01/04-01/07-3436-tENG. Situation in the Democratic Republic of the Congo in the case of the Prosecutor v. Germain Katanga, Judgment pursuant to article 74 of the Statute. 
ICC (12 June 2014). PTC I, ICC-02/11-01/11-656-Red. Situation in the Republic of Côte D'ivoire in the case of the Prosecutor v. Laurent Gbagbo, Decision on the confirmation of charges against Laurent Gbagbo.

ICC (29 January 2015). ICC-01/13. Situation on the registered vessels of The Union of The Comoros, The Hellenic Republic and The Kingdom of Cambodia, Application for Review pursuant to Article 53(3)(a) of the Prosecutor's Decision of 6 November 2014 not to initiate an investigation in the Situation.

ICC (16 July 2015). ICC-01/13. Situation on the registered vessels of The Union of The Comoros, The Hellenic Republic and The Kingdom of Cambodia, Decision on the request of the Union of the Comoros to review the Prosecutor's decision not to initiate an investigation.

Office of the Prosecutor -OTP- (2007). Draft Policy Paper on the Interests of Justice, September 2007.

OTP (2013). Draft Policy Paper on Preliminary Examinations, November 2013.

OTP (2014). Situation on Registered Vessels of Comoros, Greece and Cambodia. Article 53(1) Report, 6 November 2014.

OTP (2014a). Statement of the Prosecutor of the International Criminal Court, Fatou Bensouda, on concluding the preliminary examination of the situation referred by the Union of Comoros: "Rome Statute legal requirements have not been met". 6 November 2014 . 